\section{NORM: A subroutine for updating the norms of a reference group}

NORVAL F. POHL

Arizona State University, Tempe, Arizona 85281

and

\section{SAN-YUN W. TSAI}

California State University, Sacramento, California 95819

Angoff (1971) and Cronbach (1970) have admonished educational researchers to develop local norms for all types of measurement instruments. The development of such norms usually involves the repeated administration of tests, and thus researchers are faced with the problem of updating the norms after each administration of a measurement instrument. The widespread use of computer-scored tests would suggest that estimated parameter values or "norms" for populations of interest would be routinely updated after each administration of a measurement instrument. However, the updating algorithms that have been suggested are computationally awkward and generally require unrealistic amounts of computer mass storage and/or processing. For example, Clarke (1971) describes an algorithm for inclusion of a vector from a data set that requires access to the entire data base.

Because of the computational burdens, reference group norms are rarely updated. Thus, the typical procedure followed by educational researchers is to (1) identify a population of interest, (2) administer a measuring instrument, (3) score the results, (4) estimate the values of the parameter of interest (i.e., establish norms), and (5) assume those values hold over time. By and large, researchers are forced to interpret current behavior in light of group norms that may be quite dated. For certain types of research, group norms for selecting a particular period in time are appropriate (e.g., college students' attitudes toward "the military" during the Vietnam War). But for many types of research, a dynamic rather than a static reference group is needed (e.g., an evolving definition of personal privacy).

A relatively simple algorithm for updating the mean and variance of a data set with the addition of each new observation has been suggested by Krus (1978). This procedure has been generalized to include updating measures of skewness and kurtosis and formulated into a computer subroutine, NORM.

Program. The subroutine NORM can be used to update the values of the (1) mean, (2) variance, (3) skewness, and (4) kurtosis. Both skewness and kurtosis are measured using the moments about the mean. The updating algorithm does not require storage or manipulation of an entire data set, and it does allow for unlimited expansion of the reference group.

Input and Output. Updating can be affected after each administration of the measurement instrument, provided four values are accessible after each update: the mean, the variance, $a_{3}$ (a measure of skewness), and $a_{4}$ (a measure of kurtosis).

Language. The subroutine NORM is written in BASIC and requires only 42 statements, including comments. The mathematical calculations are relatively simple and may be easily converted to any programming language that utilizes subroutines.

Availability. A copy of the subroutine along with a flow chart with computational formulas and a data element dictionary are available without cost from Norval F. Pohl, College of Business Administration, Arizona State University, Tempe, Arizona 85281.

\section{REFERENCES}

Angoff, W. H. Scales, norms and equivalent scores. In R. L. Thorndike (Ed.), Educational measurement. Washington, D.C: American Council on Education, 1971.

ClARKE, M. R. B. Updating mean and dispersion matrix. Journal of the Royal Statistical Society, 1971, 20, 206-209.

Cronbach, L. J. Essentials of psychological testing (3rd ed.). New York: Harper \& Row, 1970.

Krus, D. J. Techniques for construction of computerized tests with automated interpretation. Proceedings of Western Educational Computing Conference, 1978, pp. 104-107.

(Accepted for publication April 18, 1980.) 